Journal of Medicinal Plants

Research Article

\title{
Phytochemical constituents of the fruits of Kelussia odoratissima Mozaff., an aromatic plant endemic to Iran
}

\author{
Mahnaz Khanavi ${ }^{1,2}$, Shima Ghadami ${ }^{1}$, Ghazal Sadaghiani-Tabrizi ${ }^{1}$, Mohammad-Reza \\ Delnavazi ${ }^{1, *}$
}

${ }^{1}$ Department of Pharmacognosy, Faculty of Pharmacy, Tehran University of Medical Sciences, Tehran, Iran

${ }^{2}$ Faculty of Land and Food Systems, University of British Columbia, Vancouver, BC, Canada

\begin{tabular}{|c|c|}
\hline ARTICLE INFO & ABSTRACT \\
\hline $\begin{array}{l}\text { Keywords: } \\
\text { Kelussia odoratissima } \\
\text { Apiaceae } \\
\text { Flavonoid } \\
\text { Essential oil } \\
\text { (Z)-Ligustilide }\end{array}$ & 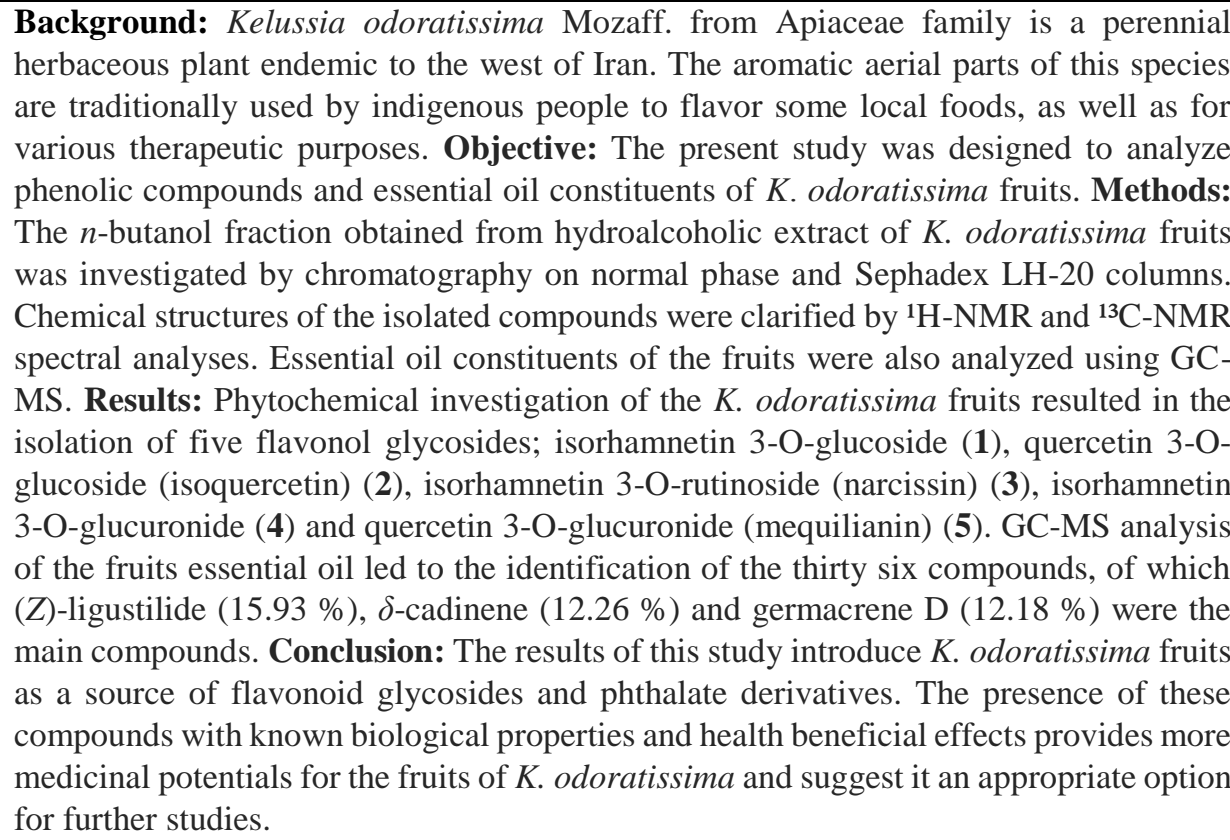 \\
\hline
\end{tabular}

\section{Introduction}

The genus Kelussia from Apiaceae family is represented by only one member, Kelussia odoratissima Mozaff., which is found in central Zagros mountains, west of Iran [1]. The aerial parts of this aromatic species are used by indigenous people under the local names of "Kellos" and "Karafse-Bakhtiari" as vegetable, as flavoring agent in yogurt, for the treatment of indigestion, rheumatism, gastric ulcer, cough,

Abbreviations: NMR, Nuclear magnetic resonance; GC-MS, Gas Chromatography-Mass Spectrometry; TLC, Thin Layer Chromatography

* Corresponding author: delnavazi@tums.ac.ir

doi: $10.52547 /$ jmp. 20.79 .1

Received 18 February 2021; Received in revised form 19 June 2021; Accepted 3 July 2021

(C) 2020. Open access. This article is distributed under the terms of the Creative Commons Attribution-NonCommercial 4.0 International License (https://creativecommons.org/licenses/by-nc/4.0/) 
pain, diabetes and as a sedative agent [2, 3]. A review on literature shows that extracts and essential oils obtained from different parts of $K$. odoratissima have been reported for their biological and pharmacological activities such as antioxidant [4, 5], antibacterial [6], larvicidal [7], antileishmanial [8], cytotoxic [9], spasmolytic [10], anti-inflammatory [11], antihypertensive [12], sedative and anxiolytic [13] effects. One study reported the isolation of two steroids, stigmasterol and $\beta$-sitosterol and one phthalide, 3-butyliden-4,5-dihydrophthalide from the $n$-hexane extract of $K$. odoratissima fruits [14]. Furthermore, they identified thirty eight compounds in the fruits essential oil, of which (Z)-ligustilide $(29.2 \%)$, germacrene B (15.9\%) and germacrene D $(15.5 \%)$ were the major compounds [14]. Another study reported the analysis of fatty acids in the fruits of $K$. odoratissima and showed that among the five fatty acids identified in the fruits oil, petroselinic acid $(72.35 \%)$ and linoleic acid $(19.14 \%)$ were the main acids [15]. In the mentioned study, $K$. odoratissima fruits were also reported as a source of phenolic compounds by a total phenolic content of 218.15 milligrams gallic acid equivalents (GAE) per gram of dry fruit weight [15]. Beside the high phenolic content, there is not any report on phenolic principles of $K$. odoratissima fruits. Therefore, the present study was designed to isolation and structural elucidation of the phenolic compounds present in the fruits of this valuable medicinal plant. The essential oil composition of the fruits was also identified and compared by related data previously reported for $K$. odoratissima.

\section{Materials and Methods}

\subsection{Plant material}

The fruits of Kelussia odoratissima Mozaff. were purchased from Pakan-Bazr Co., Isfahan,
Iran (Plant source: Fereydunshahr region, Isfahan, Iran.; Collection date: July 2017). The identity of the fruits was authenticated by botanist Dr. Yousef Ajani (Research Institute of Forest and Rangelands, Tehran, Iran) and the code of PMP-2694 was assigned for it at the herbarium of Faculty of Pharmacy, Tehran University of Medical Sciences, Tehran, Iran.

\subsection{Extraction of Phenolics}

The dried and comminuted fruits of $K$. odoratissima $(1.4 \mathrm{~kg})$ were subjected to extraction using maceration method with dichloromethane and methanol-water (70:30), successively $(4 \times 5 \mathrm{~L}$, each). The obtained extracts were concentrated using a rotary evaporator at $45^{\circ} \mathrm{C}$ under the reduced pressure and then dried in vacuum oven. Hydroalcoholic extract $(250 \mathrm{~g})$ was suspended in water $(0.5 \mathrm{~L})$ and fractionated by equal volume of $n$-butanol $(\times 3)$ to extract its phenolic compounds.

\subsection{Isolation and purification of the compounds}

A portion of the $n$-butanol fraction ( $20 \mathrm{~g}$ ) was chromatographed on a normal phase silica gel column (Mesh 230-400, Merck) using a gradient solvent mixture of $\mathrm{CHCl}_{3}-\mathrm{MeOH}$ (100:0 to 50:50) to get eighteen subfractions (B1-B18). Thin layer chromatography (Pre-coated silica gel $\mathrm{GF}_{254}$ plates, Merck) was applied to monitor column chromatography and fractions giving similar spots under UV (254 and $366 \mathrm{~nm}$ ) followed by the exposure to ammonia vapor were combined. Based on the TLC analysis, subfractions B6, B10, B11, B14 and B16 were chosen for further isolation. Column chromatography of these subfractions (B6, B10, B11, B14 and B16) on a Sephadex LH-20 column using methanol as mobile phase resulted in the isolation of compounds $\mathbf{1}(19.0 \mathrm{mg}), \mathbf{2}$ 
(13.5 mg), 3 (35.4 mg), 4 (23.8 mg) and 5 (18.1), respectively. The structures of the compounds were elucidated by ${ }^{1} \mathrm{H}-\mathrm{NMR}$ and ${ }^{13} \mathrm{C}-\mathrm{NMR}$ (Varian- INOVA, $500 \mathrm{MHz}$ for ${ }^{1} \mathrm{H}-\mathrm{NMR}$ and $125 \mathrm{MHz}$ for ${ }^{13} \mathrm{C}$-NMR) spectral analysis.

\subsection{Essential oils extraction}

The comminuted fruits $(100 \mathrm{~g})$ were subjected to hydrodistillation for 3 hours, using a Clevenger apparatus. The obtained oils were dried over anhydrous sodium sulfate and keep at $4{ }^{\circ} \mathrm{C}$ until analysis.

\subsection{GC-MS analysis}

The essential oil extracted from the fruits of K. odoratissima were analyzed on a HP- 6890 gas chromatograph with a BPX5 column (30 $\mathrm{m} \times 0.25 \mathrm{~mm}$ id, $0.25 \mu \mathrm{m}$ film thickness) equipped with HP-5973 mass detector (Ionization energy: $70 \mathrm{eV}$ ) under the following conditions; $5 \mathrm{~min}$ after injection, oven temperature was increased from $50{ }^{\circ} \mathrm{C}$ to $240{ }^{\circ} \mathrm{C}$ at a rate of $3{ }^{\circ} \mathrm{C} / \mathrm{min}$ and then reached to $300{ }^{\circ} \mathrm{C}$ at the rate of $15{ }^{\circ} \mathrm{C} / \mathrm{min}$ and hold 3 min in this temperature. Injector temperature: $250{ }^{\circ} \mathrm{C}$, detector temperature: $220{ }^{\circ} \mathrm{C}$, injection volume: $1.0 \mu \mathrm{l}$, split ratio: 1:35, carrier gas: helium (99.999\%, Flow rate: 0.5 $\mathrm{ml} / \mathrm{min}$ ). The retention indices (RIs) were calculated for all identified compounds using a homologous series of $n$-alkanes $\left(\mathrm{C}_{8}-\mathrm{C}_{24}\right)$ injected under the same conditions described for the analyzed essential oil. Identification of the compounds were carried out based on computer matching with the Wiley 275.L library, as well as by comparison of RIs and mass fragmentation patterns with those published for standard compounds [16].

\section{Results}

\subsection{Isolation and structural elucidation}

Phytochemical analysis of the fruits of $K$. odoratissima using chromatography on normal phase silica gel and Sephadex LH-20 columns resulted in the isolation of five compounds (1-5) from $n$-butanol fraction of hydroalcoholic extract. The structures of the isolated compounds were characterized as isorhamnetin 3-O- $\beta$-D-glucopyranoside (1), quercetin 3-O- $\beta$-D-glucopyranoside (isoquercetin) (2), isorhamnetin 3-O- $\beta$-D-glucopyranosyl$(6 \rightarrow 1)$ - $\alpha$-L-rhamnopyranside (isorhamnetin 3O- $\beta$-D-rutinoside, narcissin) (3), isorhamnetin 3$\mathrm{O}-\beta$-D-glucuronide (4) and quercetin $3-\mathrm{O}-\beta$-Dglucuronide (mequilianin) (5) (Fig. 1) using ${ }^{1} \mathrm{H}$ NMR and ${ }^{13} \mathrm{C}$-NMR spectral analyses, as well as by comparison with published data [17-21].

\subsubsection{Spectroscopic data of the isolated compounds}

Compound 1; Isorhamnetin 3-O- $\beta$ - Dglucopyranoside $\quad\left(\mathrm{C}_{22} \mathrm{H}_{22} \mathrm{O}_{12}\right) ; \quad$ Yellow amorphous solid; $\mathrm{R}_{f}=0.6\left(\mathrm{CHCl}_{3}-\mathrm{MeOH}, 8: 2\right)$; ${ }^{1} \mathrm{H}-\mathrm{NMR}$ (DMSO- $d_{6}, 500 \mathrm{MHz}$ ): $\delta_{\mathrm{H}} 7.93(1 \mathrm{H}, d$, $\left.J=2.0 \mathrm{~Hz}, \mathrm{H} 2^{\prime}\right), 7.50(1 \mathrm{H}, d d, J=8.5$ and 2.0 $\left.\mathrm{Hz}, \mathrm{H}^{\prime}\right), 6.93\left(1 \mathrm{H}, d, J=8.5 \mathrm{~Hz}, \mathrm{H} 5^{\prime}\right), 6.46(1 \mathrm{H}$, $d, J=2.0 \mathrm{~Hz}, \mathrm{H} 8), 6.22(1 \mathrm{H}, d, J=2.0 \mathrm{~Hz}, \mathrm{H} 6)$, $5.56\left(1 \mathrm{H}, d, J=7.4 \mathrm{~Hz}, \mathrm{H}^{\prime \prime}\right), 3.83\left(3 \mathrm{H}, s, \mathrm{OCH}_{3}\right)$, 3.1-3.7 (6H, overlapped signals, $\mathrm{H} 2 "-\mathrm{H} 6 ") .{ }^{13} \mathrm{C}-$ NMR (DMSO- $\left.d_{6}, 125 \mathrm{MHz}\right): \delta_{\mathrm{C}} 177.78$ (C4), 164.55 (C7), 161.35 (C5), 156.87 (C9), 156.73 (C2), 149.63 (C3'), 147.28 (C4'), 133.46 (C3), 122.51 (C6'), 121.58 (C1'), 115.57 (C5'), 113.87 (C2'), 104.43 (C10) , 101.18 (C1"), 99.10 (C6), 94.22 (C8), 77.76 (C5"), 76.62 (C3"), 74.63 (C2"), 70.12 $(\mathrm{C} 4 "), 60.93\left(\mathrm{C6}^{\prime \prime}\right), 56.15\left(\mathrm{OCH}_{3}\right)$ [17]. 
<smiles>COc1cc(-c2oc3cc(O)cc(O)c3c(=O)c2OC(F)(F)C(=O)O)ccc1O</smiles>

Isorhamnetin 3-O- $\beta$-D-glucopyranoside (1)<smiles>O=c1c(O[C@@H]2O[C@H](CO)[C@@H](O)[C@H](O)[C@H]2O)c(-c2ccc(O)c(O)c2)oc2cc(O)cc(O)c12</smiles>

Quercetin 3-O- $\beta$-D-glucopyranoside (2)

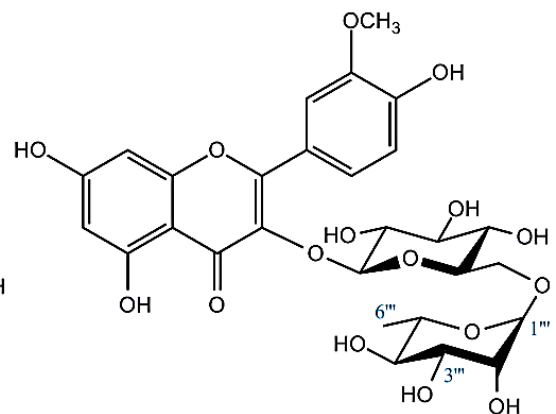

Isorhamnetin 3-O-rutinoside (3)<smiles>COc1cc(-c2oc3cc(O)cc(O)c3c(=O)c2O[C@H]2O[C@H](C(=O)O)[C@@H](O)[C@H](O)[C@H]2O)ccc1O</smiles>

Isorhamnetin 3-O-glucuronide (4)<smiles>O=C(O)C[C@H](O)[C@@H](O)[C@H](O)[C@H](O)[C@H](O)Oc1c(-c2ccc(O)c(O)c2)oc2cc(O)cc(O)c2c1=O</smiles>

Quercetin 3-O-glucoronide (5)

Fig. 1. The structures of isolated compounds (1-5) from K. odoratissima fruits

Compound 2; $\quad$ Quercetin $\left.\quad 3-O-\beta-D-\quad J=1.8 \mathrm{~Hz}, \mathrm{H} 2^{\prime}\right), 7.51(1 \mathrm{H}, d d, J=8.4$ and $1.8 \mathrm{~Hz}$, glucopyranoside (isoquercetin) $\left.\quad\left(\mathrm{C}_{21} \mathrm{H}_{20} \mathrm{O}_{12}\right) ; \quad \mathrm{H} 6^{\prime}\right), 6.91\left(1 \mathrm{H}, d, J=8.4 \mathrm{~Hz}, \mathrm{H}^{\prime}\right), 6.44(1 \mathrm{H}, d, J$ Yellow amorphous solid; $\mathrm{R}_{f}=0.5\left(\mathrm{CHCl}_{3^{-}} \quad=2.0 \mathrm{~Hz}, \mathrm{H} 8\right), 6.21(1 \mathrm{H}, d, J=2.0 \mathrm{~Hz}, \mathrm{H6}), 5.40$ $\mathrm{MeOH}, 8: 2) ;{ }^{1} \mathrm{H}-\mathrm{NMR}$ (DMSO- $\left.d_{6}, 500 \mathrm{MHz}\right): \delta_{\mathrm{H}} \quad\left(1 \mathrm{H}, d, J=7.4 \mathrm{~Hz}, \mathrm{H} 1{ }^{\prime \prime}\right), 4.40\left(1 \mathrm{H}\right.$, br $\left.s, \mathrm{H} 1^{\prime \prime \prime}\right)$, $7.58\left(1 \mathrm{H}, d, J=2.0 \mathrm{~Hz}, \mathrm{H} 2^{\prime}\right), 7.56\left(1 \mathrm{H}, d d, J=3.83\left(3 \mathrm{H}, s, \mathrm{OCH}_{3}\right), 3.0-3.7(10 \mathrm{H}\right.$, overlapped 9.0 and $\left.2.0 \mathrm{~Hz}, \mathrm{H} 6^{\prime}\right), 6.86(1 \mathrm{H}, d, J=9.0 \mathrm{~Hz}$, $\left.\mathrm{H}^{\prime}\right), 6.42(1 \mathrm{H}, d, J=2.0 \mathrm{~Hz}, \mathrm{H} 8), 6.21(1 \mathrm{H}, d, J$ $=2.0 \mathrm{~Hz}, \mathrm{H6}), 5.45(1 \mathrm{H}, d, J=7.5 \mathrm{~Hz}, \mathrm{H} 1 "), 3.1-$ $3.7(6 \mathrm{H}$, overlapped signals, $\mathrm{H} 2 "-\mathrm{H} 6 ") .{ }^{13} \mathrm{C}-$ NMR (DMSO- $\left.d_{6}, 125 \mathrm{MHz}\right): \delta_{\mathrm{C}} 177.82$ (C4), 164.52 (C7), 161.37 (C5), 156.90 (C9),156.60 (C2), $148.69\left(\mathrm{C}^{\prime}\right), 145.07\left(\mathrm{C} 3{ }^{\prime}\right), 133.80(\mathrm{C} 3)$, 122.09 (C6'), $121.63\left(\mathrm{C1}^{\prime}\right), 116.55\left(\mathrm{C} 5^{\prime}\right), 115.60$ (C2'), 104.36 (C10), 101.29 (C1"), 99.05 (C6), 94.02 (C8), 77.88 (C5"), $76.73(\mathrm{C} 3 "), 74.42$ (C2"), $70.21(\mathrm{C} 4 "), 61.26\left(\mathrm{C6}^{\prime \prime}\right)$ [18].

Compound $3 ;$ Isorhamnetin $3-O-\beta-D$ rutinoside (narcissin) $\quad\left(\mathrm{C}_{28} \mathrm{H}_{32} \mathrm{O}_{16}\right) ; \quad$ Yellow amorphous solid; $\mathrm{R}_{f}=0.3\left(\mathrm{CHCl}_{3}-\mathrm{MeOH}, 8: 2\right)$; ${ }^{1} \mathrm{H}-\mathrm{NMR}$ (DMSO- $\left.d_{6}, 500 \mathrm{MHz}\right): \delta_{\mathrm{H}} 7.83(1 \mathrm{H}, d$, signals, $\mathrm{H} 2$ "-H6" and $\left.\mathrm{H} 2{ }^{\prime \prime \prime}-\mathrm{H}-5^{\prime \prime \prime}\right), 0.94(3 \mathrm{H}, d, J$ $\left.=6.2 \mathrm{~Hz}, \mathrm{H} 6{ }^{\prime \prime \prime}\right) ;{ }^{13} \mathrm{C}-\mathrm{NMR}$ (DMSO- $d_{6}, 125$ $\mathrm{MHz}): \delta_{\mathrm{C}} 177.71(\mathrm{C} 4), 164.34(\mathrm{C} 7), 161.26(\mathrm{C} 5)$, 157.01 (C9), 156.94 (C2), 149.57 (C4'), 147.26 (C3'), 133.48 (C3), 122.77 (C6'), 121.54 (C1'), 115.57 (C5'), 113.63 (C2'), 104.44 (C10), 101.54 (C1"), 101.25 (C1"'), 99.08 (C6), 94.31 (C8), 76.54 (C5"), 76.20 (C3"), 74.52 (C2"), 72.03 (C4"'), 70.82 (C3"'), 70.57 (C2'"'), 70.39 (C4"), 68.68 (C5"'), 67.37 (C6"), $56.12\left(\mathrm{OCH}_{3}\right), 17.99$ $\left(\mathrm{C} 6^{\prime \prime \prime}\right)$ [19].

Compound 4; Isorhamnetin 3-O- $\beta$ - Dglucuronide $\left(\mathrm{C}_{22} \mathrm{H}_{20} \mathrm{O}_{13}\right)$; Yellow amorphous solid; $\mathrm{R}_{f}=0.5\left(\mathrm{CHCl}_{3}-\mathrm{MeOH}, 8: 2\right) ;{ }^{1} \mathrm{H}-\mathrm{NMR}$ (DMSO- $\left.d_{6}, 500 \mathrm{MHz}\right): \delta_{\mathrm{H}} 8.06\left(1 \mathrm{H}, b r s, \mathrm{H} 2^{\prime}\right)$, 
$7.36\left(1 \mathrm{H}, \mathrm{br} d, J=8.4, \mathrm{H}^{\prime}\right), 6.87(1 \mathrm{H}, d, J=8.4$ $\mathrm{Hz}, \mathrm{H} 5$ '), $6.16(1 \mathrm{H}, b r s, \mathrm{H} 8), 5.99(1 \mathrm{H}, b r s, \mathrm{H} 6)$, $5.57\left(1 \mathrm{H}, d, J=7.7 \mathrm{~Hz}, \mathrm{H}^{\prime \prime}\right), 3.80\left(3 \mathrm{H}, s, \mathrm{OCH}_{3}\right)$, 3.21-3.39 (4H, overlapped signals, $\mathrm{H} 2 "-\mathrm{H} 5 ") .{ }^{13} \mathrm{C}$ NMR (DMSO- $\left.d_{6}, 125 \mathrm{MHz}\right): \delta_{\mathrm{C}} 177.55$ (C4), 173.13 (C6"), 165.44 (C7), 161.21 (C5), 156.81 (C9), 156.81 (C2), 149.38 (C4'), 147.14 (C3'), 133.23 (C3), $122.24\left(\mathrm{C}^{\prime}\right), 121.68\left(\mathrm{Cl}^{\prime}\right), 115.31$ (C5'), 114.48 (C2'), 103.68 (C10), 101.28 (C1"), 99.02 (C6), 94.28 (C8), 76.42 (C3"), 76.61 (C5"), 74.35 (C2"), $72.44\left(\mathrm{C}^{\prime \prime}\right), 56.13\left(\mathrm{OCH}_{3}\right)$ [20].

Compound 5; Quercetin 3-O- $\beta-D$ glucuronide (mequilianin) $\left(\mathrm{C}_{21} \mathrm{H}_{18} \mathrm{O}_{13}\right)$; Yellow amorphous solid; $\mathrm{R}_{f}=0.4\left(\mathrm{CHCl}_{3}-\mathrm{MeOH}, 8: 2\right)$; ${ }^{1} \mathrm{H}-\mathrm{NMR}$ (DMSO- $\left.d_{6}, 500 \mathrm{MHz}\right): \delta_{\mathrm{H}} 8.23(1 \mathrm{H}, b r$ $\left.s, \mathrm{H} 2^{\prime}\right), 7.33\left(1 \mathrm{H}, d d, J=8.4\right.$ and $\left.1.2 \mathrm{~Hz}, \mathrm{H} 6^{\prime}\right)$, $6.83\left(1 \mathrm{H}, d, J=8.4 \mathrm{~Hz}, \mathrm{H} 5^{\prime}\right), 6.24(1 \mathrm{H}, b r s, \mathrm{H} 8)$, $6.06\left(1 \mathrm{H}\right.$, br s, H6), $5.15\left(1 \mathrm{H}, d, J=7.3 \mathrm{~Hz}, \mathrm{H} 1^{\prime \prime}\right)$, 3.1-3.5 (4H, overlapped signals, $\mathrm{H} 2 "-\mathrm{H} 6 ") .{ }^{13} \mathrm{C}$ NMR (DMSO- $d_{6}, 125 \mathrm{MHz}$ ): $\delta_{\mathrm{C}} 177.27$ (C-4), 172.95 (C6"), 165.06 (C7), 160.99 (C5), 157.25
(C9), 157.25 (C2), 148.62 (C4'), $145.04\left(\mathrm{C}^{\prime}\right)$, 134.35 (C3), 121.05 (C6), 121.05 (C1'), 118.10 (C-5'), 115.81 (C-2'), 103.52 (C10), 103.52 (C1"), 100.27 (C-6), 94.79 (C-8), 76.92 (C-3"), 74.54 (C-5"), 74.41 (C-2"), 72.05 (C-4") [21].

\subsection{Essential oil composition}

Hydrodistillation of the fruits of $K$. odoratissima led to the extraction of a pale yellowish oil with the yield of $1.5 \%$ (v/W). GCMS analysis of the obtained essential oil resulted in the identification of the thirty six compounds, representing $90.74 \%$ of the oil (Table 1). Among the identified compounds, $(Z)$-ligustilide (15.93 $\%), \delta$-cadinene $(12.26 \%)$ and germacrene $\mathrm{D}$ $(12.18 \%)$ were the main compounds (Fig. 2) and oxygenated sesquiterpenes with relative percentage of $53.68 \%$ were the main group of constituents identified in essential oil of $K$. odoratissima fruits.

Table 1. Chemical composition of the essential oil of $K$. odoratissima fruits obtained by hydrodistillation method

\begin{tabular}{cccc}
\hline No. & Compound name $^{\mathrm{a}}$ & $\boldsymbol{\%}$ & RI $^{\mathrm{b}}$ \\
\hline 1 & $\alpha$-Pinene & 0.09 & 939 \\
2 & $\beta$-Pinene & 0.35 & 978 \\
3 & 2-Ethyl-2-hexenal & 0.71 & 1027 \\
4 & $\beta$-Phellandrene & 3.23 & 1161 \\
5 & 5-Pentylcyclohexa-1,3-diene & 1.43 & 1170 \\
6 & Lavandulol & 0.14 & 1215 \\
7 & Acetic acid octyl ester & 0.24 & 1227 \\
8 & Citronellol & 0.27 & 1292 \\
9 & Lavandulyl acetate & 0.75 & 1374 \\
10 & Ylangene & 0.62 & 1378 \\
11 & $\alpha$-Copaene & 2.92 & 1388 \\
12 & $\beta$-Cubebene & 0.48 & 1391 \\
13 & $\beta$-Elemene & 2.04 & 1415 \\
14 & $\alpha$-Barbatene & 0.42 & 1417 \\
15 & Lavandulyl isobutyrate & 3.80 & 1435 \\
16 & $\gamma$-Elemene & 4.34 & 1437 \\
17 & Sativene & 1.36 & 1453 \\
18 & $\alpha$-Humulene & 1.09 & 1481 \\
19 & $\gamma$-Muurolene & 0.49 & 1483 \\
20 & Germacrene D & 12.18 & \\
\hline
\end{tabular}


Table 1. Chemical composition of the essential oil of $K$. odoratissima fruits obtained by hydrodistillation method (Continued)

\begin{tabular}{cccc}
\hline No. & Compound name $^{\mathbf{a}}$ & $\mathbf{\%}$ & RI $^{\mathbf{b}}$ \\
\hline 21 & Alloaromadendrene & 1.81 & 1487 \\
22 & $\gamma$-Amorphene & 1.07 & 1492 \\
23 & $\alpha$-Selinene & 1.84 & 1504 \\
24 & $\alpha$-Muurolene & 1.78 & 1512 \\
25 & $\gamma$-Cadinene & 2.80 & 1521 \\
26 & $\delta$-Cadinene & 12.26 & 1532 \\
27 & Selina-3,7(11)-diene & 2.16 & 1571 \\
28 & Neryl 2-methyl butanoate & 4.81 & 1556 \\
29 & Germacrene B & 4.02 & 1582 \\
30 & Spatulenol & 0.56 & 1599 \\
31 & Salvial-4(14)-en-1-one & 0.27 & 1648 \\
32 & $\alpha$-epi-Muurolol & 0.47 & 1658 \\
33 & 3-Butylphthalide & 0.61 & 1678 \\
34 & (Z)-Butylidene phthalide & 3.05 & 1797 \\
35 & (Z)-Ligustilide & 15.93 & 1741 \\
36 & (E)-Ligustilide & 0.35 & \\
& Monoterpene hydrocarbons & 3.67 & 9.77 \\
\end{tabular}

${ }^{\mathrm{a}}$ Compounds listed in order of elution from BPX5 column; ${ }^{\mathrm{b}}$ Retention indices to $\mathrm{C}_{8}-\mathrm{C}_{24} n$-alkanes on BPX5 column.<smiles>CCC/C=C1\OC(=O)C2=C1CCC=C2</smiles>

(Z)-Ligustilide<smiles>CC1=CC2C(=C(C)CCC2C(C)C)CC1</smiles>

$\delta$-Cadinene<smiles>C=C1C=CC(C(C)C)CCC=C(C)CC1</smiles>

Germacrene D

Fig. 2. The structures of main compounds identified in essential oil of $K$. odoratissima fruits

\section{Discussion}

Five flavonol 3-O-glycosides, namely, isorhamnetin 3-O- $\beta$-D-glucopyranoside (1), quercetin 3-O- $\beta$-D-glucopyranoside (isoquercetin) (2), isorhamnetin 3-O- $\beta$-D-rutinoside (narcissin) (3), isorhamnetin 3-O- $\beta$-D-glucuronide (4) and quercetin 3-O- $\beta$-D-glucuronide (mequilianin) (5) were isolated from the fruits of $K$. odoratissima in present study for the first time.

Journal of Medicinal Plants
Previously, two steroid derivatives, stigmasterol and $\beta$-sitosterol and one phthalide, 3-butyliden-4,5-dihydrophthalide reported from $n$-hexane extract of $K$. odoratissima fruits [14]. Flavonoids are phenolic compounds with wellknown free radical scavenging activity which makes them appropriate food supplements to prevent oxidative stress related diseases such as cancers, diabetes, cardiovascular, inflammatory 
and neurodegenerative diseases (such as Alzheimer and Parkinson) [22].

The potent antioxidant activity of isorhamnetin 3-O- $\beta$-D-rutinoside, a major flavonol 3-O-glycoside isolated in present study, has been reported in DPPH $\left(\mathrm{IC}_{50} ; 9.01 \mu \mathrm{M}, \mathrm{IC}_{50}\right.$ value of ascorbic acid as positive control; 11.93 $\mu \mathrm{M})$ and $\mathrm{ONOO}^{-}\left(\mathrm{IC}_{50} ; 2.56 \mu \mathrm{M}, \mathrm{IC}_{50}\right.$ value of DL-penicillamin as positive control; $5.1 \mu \mathrm{M}$ ) methods [23]. This compound (isorhamnetin 3O- $\beta$-D-rutinoside) has also been reported as one of antimicrobial principles of Atriplex halimus aerial parts with considerable antibacterial activity against Streptococcus pyogenes (inhibition zone; $17 \pm 0.09 \mathrm{~mm}$ ), Escherichia coli (inhibition zone; $16 \pm 0.09 \mathrm{~mm}$ ) and Acinetobacter baumanii (inhibition zone; $16 \pm$ $0.13 \mathrm{~mm}$ ), having a low cytotoxicity on PMNCs $\left(\mathrm{IC}_{50} ; 450 \mu \mathrm{g} \mathrm{ml}^{-1}\right)$ [24].

Isorhamnetin 3-O- $\beta$-D-glucopyranoside and quercetin 3-O- $\beta$-D-glucopyranoside (isoquercetin), two other flavonol glycosides isolated from $K$. odoratissima fruits in present study, have been documented for its antioxidant [25], antidiabetic [26] and hepatoprotective [27] activities. Furthermore, in a bioassay-guided fractionation study quercetin 3-O-glucoside was isolated as active compounds of Sambucus ebulus L. leaves with remarkable wound healing activity [28].

Isorhamnetin and quercetin were also isolated as their 3-O- $\beta$-D-glucuronide derivatives in the present study. A bioactivity-guided isolation study reported the isolation of isorhamnetin 3-O$\beta$-D-glucuronide as one of active compounds of Sanguisorba officinalis L. (Rosaceae) with considerable lipid accumulation inhibition on 3T3-L1 cells ( $\left.\mathrm{IC}_{30} ; 18.43 \mu \mathrm{M}\right)$ [29]. Chuquiraga spinosa (Asteraceae), Cichorium spinosum L. (Asteraceae), Foeniculum vulgare L. (Apiaceae) and some cultivars of Vitis vinifera L. (Vitaceae) are examples of plants reported to contain isorhamnetin 3-O- $\beta$-D-glucuronide [30-34].
Quercetin 3-O- $\beta$-D-glucuronide has been found in some plant species such as Calligonum comosum L. (Polygonaceae), Rubus ulmifolius Schott. (Rosaceae) and Phaseolus vulgaris L. (Fabaceae), as well as one of major human metabolites of quercetin [35-38]. One study suggested that supplementation of quercetin 3-O$\beta$-D-glucuronide can reduce the relative risk for developing Alzheimer's disease (AD) dementia [39]. They showed that quercetin $3-O-\beta$-Dglucuronide can significantly reduce the generation of $\beta$-amyloid $(\mathrm{A} \beta)$ peptides and improves AD-type deficits in hippocampal formation basal synaptic transmission and longterm potentiation, possibly through mechanisms involving the activation of the c-Jun N-terminal kinases and the mitogen-activated protein kinase signaling pathways [39]. In another study, quercetin 3-O- $\beta$-D-glucuronide showed to possess anti-neuroinflammatory activity in lipopolysaccharide (LPS)-induced inflammation in BV2 cells by inhibition of the production of NO and PGE2 and reducing the levels of some pro-inflammatory cytokines such as TNF- $\alpha$ and interlukin-1 $\beta$ [40]. Moreover, beneficial effects of quercetin 3-O- $\beta$-D-glucuronide in arteriosclerosis prevention has been reported via elevating plasma HDLC followed by induction of ABCA1 (ATP-binding cassette, subfamily A, member 1) expression, a crucial cholesterol transporter involved in reverse cholesterol transport [41].

In the present study, GC-MS analysis of the essential oil extracted from the fruits of $K$. odoratissima resulted in the identification of thirty six compounds, of which (Z)-ligustilide $(15.93 \%), \delta$-cadinene $(12.26 \%)$ and germacrene D $(12.18 \%)$ were the main compounds (Fig. 2). One study reported $\alpha$-caryophyllene $(22.60 \%)$, $\alpha$-humulene $(20.0 \%)$ and cyclopropane $(11.54$ $\%)$ as the main compounds of the essential oil of $K$. odoratissima fruits, gathered from Zardkooh 
Mountain, (Charmahal-Bakhtiari, Iran) [42]. In another study on the fruits of this plant collected from Samsami region (Chaharmahal-Bakhtiari, Iran) (Z)-Ligustilide (86.0\%), (2E)-decen-1-ol $(8.0 \%)$, pentyl cyclohexa-1,3-diene $(4.4 \%)$ were characterized as main compounds of its essential oil [43]. The results of previous reports on essential oil composition of different parts of $K$. odoratissima have been summarized in Table 2 [5, 7, 8, 13, 15, 43-47]. Beside possible genetic diversity, variation observed in essential oil constituents may be raised from some geographical differences between the populations of $K$. odoratissima [48].

Table 2. The results of essential oil analysis of $K$. odoratissima from the previous and present studies.

\begin{tabular}{|c|c|c|c|c|c|}
\hline Location of collection & Date & Method & Part & Main compounds (\%) & Ref. \\
\hline $\begin{array}{l}\text { Fereydunshahr, Isfahan, } \\
\text { Iran }\end{array}$ & $\begin{array}{c}\text { Dec } \\
2018\end{array}$ & $\mathrm{HD}^{\mathrm{a}}$ & Fruit & $\begin{array}{c}\text { (Z)-Ligustilide }(15.9 \%), \delta \text {-Cadinene }(12.3 \%) \text {, } \\
\text { Germacrene D }(12.2 \%)\end{array}$ & $\begin{array}{c}\text { Present } \\
\text { study }\end{array}$ \\
\hline $\begin{array}{l}\text { Dishmook, Kohgiluye- } \\
\text { Buyer Ahmad, Iran }\end{array}$ & \multirow{3}{*}{$\begin{array}{l}\text { May } \\
2014\end{array}$} & \multirow{3}{*}{ HD } & \multirow{3}{*}{ Leaf } & $\begin{array}{c}\text { (Z)-Ligustilide (58.7\%), Carvacrol }(7.8 \%) \text {, } \\
\text { trans-3-Butylidene phthalide (4.9\%), Thymol } \\
(4.5 \%)\end{array}$ & \multirow{3}{*}{5} \\
\hline $\begin{array}{l}\text { Keloseh region, Isfahan, } \\
\text { Iran }\end{array}$ & & & & $\begin{array}{c}\text { (Z)-Ligustilide }(53.5 \%) \text {, Thymol }(7.9 \%) \text {, trans- } \\
\text { 3-Butylidene phthalide }(3.3 \%)\end{array}$ & \\
\hline $\begin{array}{l}\text { Fereydunshahr, Isfahan, } \\
\text { Iran }\end{array}$ & & & & $\begin{array}{c}\text { (Z)-Ligustilide (51.3\%), Thymol (8.7\%), } \\
\text { Carvacrol }(3.2 \%)\end{array}$ & \\
\hline $\begin{array}{l}\text { Central Zagros Mountain, } \\
\text { Chaharmahal-Bakhtiari, } \\
\text { Iran } \\
\end{array}$ & $\begin{array}{c}\text { Apr } \\
2009\end{array}$ & HD & Leaf & $\begin{array}{c}\text { (Z)-Ligustilide }(77.7 \%), 2 \text {-Octen-1-ol acetate } \\
(6.3 \%),(E) \text {-Ligustilide }(2.3 \%)\end{array}$ & 7 \\
\hline $\begin{array}{l}\text { Kohgiluye-Buyer Ahmad, } \\
\text { Iran }\end{array}$ & $\begin{array}{l}\text { Apr- } \\
\text { May } \\
2012\end{array}$ & HD & $\begin{array}{c}\text { Aerial } \\
\text { part }\end{array}$ & $\begin{array}{c}\text { (Z)-Ligustilide (34.5\%), (E)-Ligustilide (11.8\%), } \\
\text { 3-(Z)-Butylidenephthalide (8.8\%), Dec-9-en-1- } \\
\text { ol (5.9\%) }\end{array}$ & 8 \\
\hline $\begin{array}{l}\text { Central Zagros mountain, } \\
\text { Iran }\end{array}$ & $\begin{array}{l}\text { Mar } \\
2006\end{array}$ & HD & $\begin{array}{c}\text { Aerial } \\
\text { part }\end{array}$ & $\begin{array}{c}\text { (Z)-Ligustilide }(85.9 \%), \alpha \text {-Copaene }(1.4 \%), \delta \text { - } \\
\text { Cadinene }(0.7 \%)\end{array}$ & 13 \\
\hline $\begin{array}{c}\text { Fereydunshahr, Isfahan, } \\
\text { Iran }\end{array}$ & $\begin{array}{l}\text { Aug } \\
2009\end{array}$ & HD & Fruit & $\begin{array}{l}Z \text { Z-ligustilide }(51.0 \%), \delta \text {-Terpinen-7-al }(10.3 \%), \\
\delta \text {-terpinene }(5.3 \%), \text { Cumin aldehyde }(5.2 \%)\end{array}$ & 15 \\
\hline $\begin{array}{l}\text { Zardkooh Mountain, } \\
\text { Charmahal-Bakhtiari, Iran }\end{array}$ & $\begin{array}{c}\text { Jul-Aug } \\
2011\end{array}$ & HD & Fruits & $\begin{array}{c}\alpha \text {-Caryophyllene (22.6\%), } \alpha \text {-Humulene (20.0\%), } \\
\text { Cyclopropane }(11.5 \%)\end{array}$ & 42 \\
\hline \multirow{5}{*}{$\begin{array}{l}\text { Samsami region, } \\
\text { Chaharmahal-Bakhtiari, } \\
\text { Iran }\end{array}$} & \multirow{5}{*}{$\begin{array}{c}\text { Jun-Jul } \\
2014\end{array}$} & \multirow{5}{*}{ HD } & Root & $\begin{array}{c}\text { (Z)-Ligustilide }(54.0 \%),(2 E) \text {-Decen-1-ol } \\
\text { (10.7\%), Pentyl cyclohexa-1,3-diene }(6.4 \%) \text {, } \\
\text { (3Z)-Butylidene phthalide }(5.8 \%)\end{array}$ & \multirow{5}{*}{43} \\
\hline & & & Stem & $\begin{array}{c}\text { (Z)-Ligustilide (58.7\%), (2E)-Decen-1-ol } \\
(11.6 \%), \text { Pentyl cyclohexa-1,3-diene }(4.4 \%)\end{array}$ & \\
\hline & & & Leaf & $\begin{array}{l}\text { (Z)-Ligustilide }(66.8 \%),(2 E) \text {-Decen-1-ol } \\
(12.3 \%), \text { Pentyl cyclohexa-1,3-diene }(3.8 \%)\end{array}$ & \\
\hline & & & Flower & $\begin{array}{l}\text { (Z)-Ligustilide }(62.4 \%) \text {, Geranyl butyrate } \\
(9.0 \%) \text {, trans-Muurola-4(14) 5-diene }(5.5 \%)\end{array}$ & \\
\hline & & & Fruit & $\begin{array}{l}\text { (Z)-Ligustilide }(86.0 \%),(2 E) \text {-Decen-1-ol } \\
(8.0 \%), \text { Pentyl cyclohexa-1,3-diene }(4.4 \%)\end{array}$ & \\
\hline
\end{tabular}


Table 2. The results of essential oil analysis of $K$. odoratissima from the previous and present studies (Continued)

\begin{tabular}{|c|c|c|c|c|c|}
\hline Location of collection & Date & Method & Part & Main compounds (\%) & Ref. \\
\hline \multirow{3}{*}{$\begin{array}{l}\text { Keloseh region, } \\
\text { Fereydunshahr, } \\
\text { Isfahan, Iran }\end{array}$} & \multirow{3}{*}{$\begin{array}{l}\text { Aug } \\
2007\end{array}$} & \multirow{3}{*}{ HD } & Stem & $\begin{array}{c}\text { Borneol (36.9 \%), Bornyl acetate }(14.0 \%) \text {, } \\
\text { 1,8-Cineol }(13.6 \%) \text {, Camphor }(9.5 \%)\end{array}$ & \multirow{3}{*}{44} \\
\hline & & & Flower & $\begin{array}{l}\text { 1,8-Cineol (22.0\%), Camphor (20.1\%), } \alpha \text {-Pinene } \\
(19.0 \%), \text { Camphene (12.0\%), Bornyl acetate }(5.8 \%)\end{array}$ & \\
\hline & & & Leaf & $\begin{array}{l}\beta \text {-Terpinene }(23.0 \%), \text { Sabinene }(9.0 \%), \alpha \text {-Thujene } \\
(8.4 \%)\end{array}$ & \\
\hline $\begin{array}{l}\text { Fereydunshahr, } \\
\text { Isfahan, Iran }\end{array}$ & Jul 2007 & HD & $\begin{array}{c}\text { Aerial } \\
\text { part }\end{array}$ & $\begin{array}{c}\text { (Z)-Ligustilide }(87.6 \%),(E) \text {-Ligustilide }(3.2 \%), \\
\text { Piperitone epoxide }(3.1 \%)\end{array}$ & 45 \\
\hline $\begin{array}{l}\text { Bazoft region, } \\
\text { Chaharmahal- } \\
\text { Bakhtiari, Iran }\end{array}$ & \multirow{3}{*}{$\begin{array}{l}\text { April } \\
2008\end{array}$} & \multirow{3}{*}{ HD } & \multirow{3}{*}{$\begin{array}{c}\text { Aerial } \\
\text { part }\end{array}$} & $\begin{array}{c}\text { (Z)-Ligustilide }(47.3 \%),(3 E) \text {-Butyldiene phthalide } \\
(17.38 \%),(E) \text {-Ligustilide }(6.3 \%), 2 \text {-Octen-1-ol } \\
\text { acetate }(5.4 \%)\end{array}$ & \multirow{3}{*}{46} \\
\hline $\begin{array}{l}\text { Koohrang region, } \\
\text { Chaharmahal- } \\
\text { Bakhtiari, Iran }\end{array}$ & & & & $\begin{array}{c}\text { (Z)-Ligustilide (33.7\%), (3E)-Butyldiene phthalide } \\
(20.1 \%),(E) \text {-Ligustilide }(6.6 \%), 2 \text {-Octen-1-ol acetate } \\
(5.2 \%)\end{array}$ & \\
\hline $\begin{array}{l}\text { Samsami region, } \\
\text { Chaharmahal- } \\
\text { Bakhtiari, Iran }\end{array}$ & & & & $\begin{array}{l}\text { (Z)-Ligustilide (37.6\%), (3E)-Butyldiene phthalide } \\
(19.9 \%),(E) \text {-Ligustilide }(7.0 \%), \text { Kessane }(5.3 \%)\end{array}$ & \\
\hline Zagros mountain, Iran & $\begin{array}{c}\text { May-Jun } \\
2012\end{array}$ & HD & $\begin{array}{c}\text { Aerial } \\
\text { part }\end{array}$ & $\begin{array}{c}\alpha \text {-Pinene (20.1\%), 1,8-Cineole (18.2\%), (Z)- } \\
\text { Ligustilide }(15.5 \%)\end{array}$ & 47 \\
\hline
\end{tabular}

(Z)-Ligustilide, a phthalide derivative identified as major constituent in most of previously studied $K$. odoratissima essential oils, has received attention for its interesting pharmacological and biological effects such as neuroprotective, anti-oxidation, antiinflammatory, analgesic and anticancer effects [49].

\section{Conclusion}

The presence of flavonoid glycosides (1-5) and phthalide derivatives with known health beneficial effects make the fruits of $K$. odoratissima as a natural remedy with valuable therapeutic potentials and suggest it an appropriate option for further studies. Meanwhile, restricted distribution of $K$. odoratissima underline the importance of an appropriate conservation approach for the uses of this species for medicinal and food purposes.

\section{Author contributions}

MK: Study supervision and data interpretation; SG: Experimental analysis and preparation of manuscript draft; GS: Experimental analysis; MD: Original idea presentation, study design, study supervision, data interpretation and revision of the manuscript.

\section{Conflict of interest}

The authors declare that there is no conflict of interest.

\section{Acknowledgment}

This research was supported by Tehran University of Medical Sciences and Health Services grant (No. 48353). 


\section{References}

1. Mozaffarian V. Flora of Iran, No.54: Umbelliferae. Tehran: Publication of Research Institute of Forests and Rangelands; 2007.

2. Amiri MS and Joharchi MR. Ethnobotanical knowledge of Apiaceae family in Iran: a review. Avicenna J. Phytomed. 2016; 6(6): 621-35. doi: 10.22038/AJP.2016.6696.

3. Ahmadi K, Omidi H, Amini Dehaghi $M$ and Naghdi Badi H. A review on the botanical, phytochemical and pharmacological characteristics of Kelussia odoratissima Mozaff. J. Med. Plants 2020; 18(72): 30-45.

4. Ahmadi F, Kadivar $M$ and Shahedi $M$. Antioxidant activity of Kelussia odoratissima Mozaff. in model and food systems. Food Chem. 2007; 105(1): 57-64. doi: 10.1016/j.foodchem. 2007.03.056.

5. Akbarian A, Rahimmalek M, Sabzalian MR and Sarfaraz D. Variation in essential oil composition, phenolic, flavonoid and antioxidant activity of Kelussia odoratissima Mozaff based on three model systems. J. Appl. Res. Med. Aromat. Plants 2019; 13: 100208. doi: 10.1016/j.jarmap.2019.100208.

6. Sureshjani MH, Yazdi FT, Mortazavi SA, Behbahani BA and Shahidi F. Antimicrobial effects of Kelussia odoratissima extracts against food borne and food spoilage bacteria "in vitro". Arch. Adv. Biosci. 2014; 5(2): 115-20. doi:10.22037/JPS.V5I2.5943.

7. Vatandoost H, Sanei Dehkordi A, Sadeghi SMT, Davari B, Karimian F, Abai MR and Sedaghat MM. Identification of chemical constituents and larvicidal activity of Kelussia odoratissima Mozaffarian essential oil against two mosquito vectors Anopheles stephensi and Culex pipiens (Diptera: Culicidae). Exp. Parasitol. 2012; 132(4): 470-574. doi: 10.1016/ j.exppara.2012.09.010.

Journal of Medicinal Plants
8. Kheirabadi KP, Dehkordi SS and Kheibari P. Effect of Kelussia odoratissima Mozaff. essential oil on promastigot form of Leishmania major (in vitro). J. HerbMed Pharmacol. 2015; 4(1): 10-14.

9. Karimian H, Arya A, Fadaeinasab M, Razavi M, Hajrezaei M, Khan AK, Ali HM, Abdulla MA and Noordin MI. Kelussia odoratissima Mozaff. activates intrinsic pathway of apoptosis in breast cancer cells associated with $\mathrm{S}$ phase cell cycle arrest via involvement of $\mathrm{p} 21 / \mathrm{p} 27$ in vitro and in vivo. Drug Des. Devel. Ther. 2017; 11: 337-50. doi: $10.2147 /$ dddt.s121518.

10. Sedighi M, Rafieian-Kopaei M, NooriAhmadabadi M. Kelussia odoratissima Mozaffarian inhibits ileum contractions through voltage dependent and beta adrenergic receptors. Life Sci. J. 2012; 9(4): 1033-8.

11. Minaiyan M, Sajjadi SE, Naderi $N$ and Taheri D. Anti-inflammatory effect of Kelussia odoratissima Mozaff. hydroalcoholic extract on acetic acid-induced acute colitis in rats. J. Rep. Pharm. Sci. 2014; 3(1): 28-35.

12. Safaeian L, Sajjadi SE, Javanmard SH and Gholamzadeh H. Antihypertensive and antioxidant effects of hydroalcoholic extract from the aerial parts of Kelussia odoratissima Mozaff. in dexamethasone-induced hypertensive rats. Adv. Biomed. Res. 2016; 5: 25. doi: 10.4103/2277-9175.176342.

13. Rabbani M, Sajjadi SE and Sadeghi M. Chemical composition of the essential oil from Kelussia odoratissima Mozaff. and the evaluation of its sedative and anxiolytic effects in mice. Clinics. 2011; 66(5): 843-8. doi: 10.1590 /s1807 -59322011000500022.

14. Sajjadi S, Shokoohinia Y and Mehramiri P. Isolation and characterization of steroids, phthalide and essential oil of the fruits of 
Kelussia odoratissima Mozaff., an endemic mountain celery. Res. Pharm. Sci. 2013; 8(1): 3541.

15. Saeedi KA and Omidbaigi R. Chemical characteristics of the seed of Iranian endemic plant Kelussia odoratissima. Chem. Nat. Compd. 2010; 46(5): 813-5. doi: 10.1007/s10600-0109754-7

16. Adams RP. Identification of essential oil components by gas chromatography/mass spectrometry. Carol Stream: Allured Publishing Corporation; 2007.

17. Aliotta G, Della Greca M, Monaco P, Pinto G, Pollio A and Previtera L. In vitro algal growth inhibition by phytotoxins of Typha latifolia L. J. Chem. Ecol. 1990; 16(9): 2637-46. doi: 10.1007/bf00988075.

18. Eldahshan OA. Isolation and structure elucidation of phenolic compounds of carob leaves grown in Egypt. Curr. Res. J. Biol. Sci. 2011; 3(1): 52-5.

19. Güvenalp Z, Özbek H, Ünsalar T, Kazaz C, Demirezer LÖ. Iridoid, flavonoid, and phenylethanoid glycosides from Wiedemannia orientalis. Turk. J. Chem. 2006; 30(3): 391-400.

20. Im SH, Wang Z, Lim SS, Lee OH and Kang IJ. Bioactivity-guided isolation and identification of anti-adipogenic compounds from Sanguisorba officinalis. Pharm. Biol. 2017; 55(1): 2057-64. doi: 10.1080/13880209.2017.1357736.

21. Nugroho A, Song BM, Lee KT, Park HJ. Quantification of antidepressant miquelianin in mature and immature fruits of Korean Rubus species. Nat. Prod. Sci. 2014; 20(4): 258-61. doi: 10.1080/13880209.2017.1357736.

22. Shahidi F and Ambigaipalan P. Phenolics and polyphenolics in foods, beverages and spices: Antioxidant activity and health effects- a review. J. Funct. Food. 2015; 18: 820-97. doi: 10.1016/j.jff.2015.06.018.
23. Hyun SK, Jung YJ, Chung HY, Jung HA, Choi JS. Isorhamnetin glycosides with free radical and $\mathrm{ONOO}^{-}$scavenging activities from the stamens of Nelumbo nucifera. Arch. Pharm. Res. 2006; 29(4): 287-92. doi: 10.1007/bf02968572.

24. El-Aasr M, Kabbash A, El-Seoud KAA, AlMadboly LA and Ikeda T. Antimicrobial and Immunomodulatory activities of flavonol glycosides isolated from Atriplex halimus L. herb. J. Pharm. Sci. Res. 2016; 8(10): 1159-68.

25. Zheng Y-Z, Deng G, Liang Q, Chen D-F, Guo R and Lai R-C. Antioxidant activity of quercetin and its glucosides from propolis: A theoretical study. Sci. Rep. 2017; 7(1): 1-11. doi: 10.1038/s41598-017-08024-8.

26. Panda $S$ and Kar A. Antidiabetic and antioxidative effects of Annona squamosa leaves are possibly mediated through quercetin-3-Oglucoside. Biofactors 2007; 31(3-4): 201-10. doi: 10.1002/biof.5520310307.

27. Lee S, Lee J, Lee $H$ and Sung J. Relative protective activities of quercetin, quercetin-3glucoside, and rutin in alcohol-induced liver injury. J. Food Biochem. 2019; 43(11): e13002. doi: 10.1111/jfbc.13002.

28. Süntar IP, Akkol EK, Yalçın FN, Koca U, Keleş $\mathrm{H}$ and Yesilada E. Wound healing potential of Sambucus ebulus L. leaves and isolation of an active component, quercetin 3-Oglucoside. J. Ethnopharmacol. 2010; 129(1): 106-14. doi: 10.1016/j.jep.2010.01.051.

29. Im SH, Wang Z, Lim SS, Lee $\mathrm{OH}$ and Kang IJ. Bioactivity-guided isolation and identification of anti-adipogenic compounds from Sanguisorba officinalis. Pharm. Biol. 2017; 55(1): 2057-64. doi: 10.1080/13880209.2017.1357736.

30. Landa A, Casado $R$ and Calvo MI. Identification and quantification of flavonoids from Chuquiraga spinosa (Asteraceae). Nat. 
Prod. Commun. 2009; 4(10): 1353-1355. doi: 10.1177/1934578X0900401008.

31. Petropoulos SA, Fernandes Â, Vasileios A, Ntatsi G, Barros L and Ferreira IC. Chemical composition and antioxidant activity of Cichorium spinosum L. leaves in relation to developmental stage. Food Chem. 2018; 239: 946-52. doi: 10.1016/j.foodchem.2017.07.043.

32. Parejo I, Jauregui O, Sánchez-Rabaneda F, Viladomat F, Bastida J and Codina C. Separation and characterization of phenolic compounds in fennel (Foeniculum vulgare) using liquid chromatography- negative electrospray ionization tandem mass spectrometry. J. Agric. Food Chem. 2004; 52(12): 3679-87. doi: 10.1021/jf030813h.

33. Castillo-Muñoz N, Gómez-Alonso S, García-Romero E and Hermosín-Gutiérrez I. Flavonol profiles of Vitis vinifera white grape cultivars. J. Food Compost. Anal. 2010; 23(7): 699-705. doi: 10.1016/j.jfca.2010.03.017.

34. Del-Castillo-Alonso MÁ, Monforte L, Tomás-Las-Heras $\mathrm{R}$, Martínez-Abaigar $\mathrm{J}$ and Núñez-Olivera E. Phenolic characteristics acquired by berry skins of Vitis vinifera cv. Tempranillo in response to close-to-ambient solar ultraviolet radiation are mostly reflected in the resulting wines. J. Sci. Food Agric. 2020; 100(1): 401-9. doi: 10.1002/jsfa.10068.

35. Badria FA, Ameen $M$ and Akl MR. Evaluation of cytotoxic compounds from Calligonum comosum L. growing in Egypt. $Z$. Naturforsch. C. 2007; 62(9-10): 656-60. doi: 10.1515/znc-2007-9-1005.

36. Dall'Acqua S, Cervellati R, Loi $\mathrm{MC}$ and Innocenti G. Evaluation of in vitro antioxidant properties of some traditional Sardinian medicinal plants: Investigation of the high antioxidant capacity of Rubus ulmifolius. Food Chem. 2008; 106(2): 745-9. doi: 10.1016/j.foodchem.2007.06.055.
37. Plumb GW, Price KR and Williamson G. Antioxidant properties of flavonol glycosides from green beans. Redox Rep. 1999; 4(3): 123-7. doi: 10.1179/135100099101534800.

38. Moon J-H, Tsushida $T$, Nakahara $K$ and Terao J. Identification of quercetin $3-\mathrm{O}-\beta$-Dglucuronide as an antioxidative metabolite in rat plasma after oral administration of quercetin. Free Radical Bio. Med. 2001; 30(11): 1274-85. doi: 10.1016/s0891-5849(01)00522-6.

39. Ho L, Ferruzzi MG, Janle EM, Wang J, Gong B, Chen TY, Lobo J, Cooper B, Wu QL, Talcott ST, Percival SS, Simon JE and Pasinetti GM. Identification of brain-targeted bioactive dietary quercetin-3-O-glucuronide as a novel intervention for Alzheimer's disease. FASEB $J$. 2013; 27(2): 769-81. doi: 10.1096/fj.12-212118. 40. Yoon CS, Kim DC, Ko WM, Kim KS, Lee DS, Kim DS, Cho HK, Seo J, Kim SY, Oh H and Kim YC. Anti-neuroinflammatory effects of quercetin-3-O-glucuronide isolated from the leaf of Vitis labruscana on LPS-induced neuroinflammation in BV2 cells. Korean $J$. Pharmacog. 2014; 45(1): 17-22.

41. Ohara K, Wakabayashi $H$, Taniguchi $Y$, Shindo K, Yajima H and Yoshida A. Quercetin3-O-glucuronide induces ABCA1 expression by LXR $\alpha$ activation in murine macrophages. Biochem. Bioph. Res. Co. 2013; 441(4): 929-34. doi: 10.1016/j.bbrc.2013.10.168.

42. Mahmoudi R, Kosari M, Zare P and Barati S. Kelussia odoratissima essential oil: biochemical analysis and antibacterial properties against pathogenic and probiotic bacteria. $J$. Agroaliment. Processes Technol. 2014; 20(1): 109-15. doi: 10.26226/morressier. 5f4ccc57648d3d8b3f362be9.

43. Raeisi S, Mirjalili MH, Nadjafi F and Hadian J. Variability in the essential oil content and composition in different plant organs of Kelussia odoratissima Mozaff. (Apiaceae) growing wild 
in Iran. J. Essent. Oil Res. 2015; 27(4): 283-8. doi: 10.1080/10412905.2015.1025917.

44. Esmaeili A, Sharafian S, Mirian S and Larijani K. Chemical composition of essential oil from leaves, stems and flowers of Kelussia odoratissima Mozaff. grown in Iran. J. Essent. Oil-Bear. Plants. 2011; 14(5): 643-6. doi: 10.1080/0972060x.2011.10643984.

45. Omidbaigi R, Sefidkon F and Saeedi K. Essential oil content and composition of Kelussia odoratissima Mozaff. as an Iranian endemic plant. J. Essent. Oil-Bear. Plants. 2008; 11(6): 594-7. doi: 10.1080/0972060X.2008.10643672.

46. Shojaei ZA, Ebrahimi A and Salimi M. Chemical composition of three ecotypes of wild celery (Kelussia odoratissima). J. Herbs Spices Med. Plants. 2011; 17(1): 62-8. doi: 10.1080/ 10496475.2011. 560089.

47. Pirbalouti AG, Sedaghat L, Hamedi B and Tirgir F. Chemical composition and antioxidant activity of essential oils of three endemic medicinal plants of Iran. Bangladesh J. Bot. 2013; 42(2): 327-32. doi: 10.3329/bjb.v42i2. 18038.

48. Baser KHC and Buchbauer G. Handbook of essential oils: science, technology, and applications: CRC press; 2015.

49. Xie Q, Zhang L, Xie L, Zheng Y, Liu K, Tang H, Liao Y and Li X. Z-Ligustilide: a review of its pharmacokinetics and pharmacology. Phytotherapy Res. 2020; 34(8): 1966-1991. doi: 10.1002/ptr.6662.

How to cite this article: Khanavi M, Ghadami Sh, Sadaghiani-Tabrizi Gh, Delnavazi MR. Phytochemical constituents of the fruits of Kelussia odoratissima Mozaff., an aromatic plant endemic to Iran. Journal of Medicinal Plants 2021; 20(79): 1-13. doi: $10.52547 /$ jmp.20.79.1 


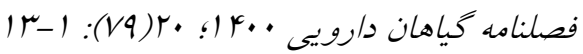

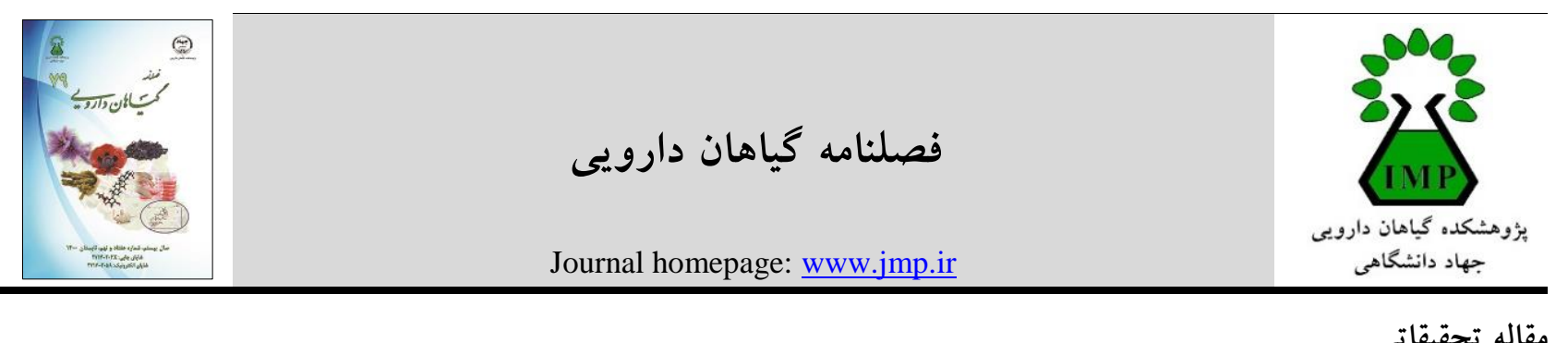

مقاله تحقيقاتى تركيبات فيتوشيميايى ميوه كلوس (Kelussia odoratissima Mozaff.)، گياهى معطر و انحصارى

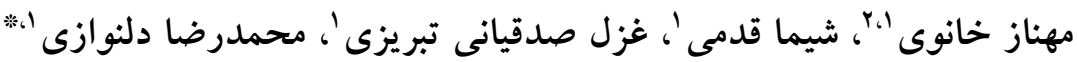

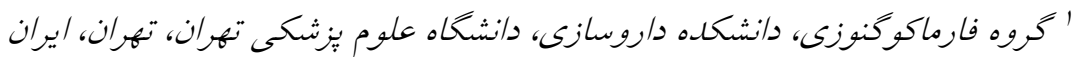

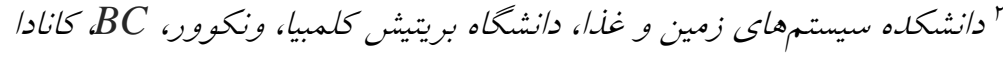

\begin{tabular}{|c|c|}
\hline جكيده & اطلاعات مقاله \\
\hline مقدمه: كَياه كلوس (Kelussia odoratissima Mozaff.) از تيره گتريان، كياهى است علفى و بايا كه به صورت & 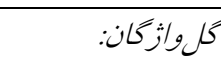 \\
\hline انحصارى در غرب ايران مىرويد. مردم بومى از بخشهاى هوايى معطر اين گياه به صورت سنتى به عنوان عطر و & كلوس \\
\hline طعمدهنده در تهيه بعضى غذاهاى محلى و همجنين در درمان بيمارىهاى مختلف استفاده مى كنند. هدف: مطالعه & تيره هتريان \\
\hline حاضر با هدف جداسازى و شـناسايى تركيبات فنولى و همجنين آناليز تركيبات اسـانس ميوههاى گياه كلوس طراحى & 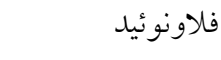 \\
\hline شده است. روش بررسى: تركيبات فراكسيون بوتانولى نرمال بدست آمده از عصاره هيدروالكلى ميوه گياه با استفاده & اسانس \\
\hline از كروماتوكرافى روى ستونهاى فاز نرمال و سفادكس ال اجج-·r مورد جداسازى و خالصسازى قرار گرفت. & 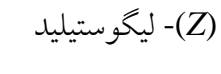 \\
\hline تركيبات اسانس بدست آمده از نمونه نيز با استفاده از تكنيك كروماتوكرافى كازى متصل به طيفسنج جرمى آناليز & \\
\hline شد. نتايج: مطالعه فيتوشـيميايى روى ميوههاى كياه كلوس به جداسازى و شناسايى بنج كليكوزيد فلاونولى با نامهاى & \\
\hline ايزورامنتين r-أُ- كلوكوزيد (1)، كوئرستين r-أ-- كلوكوزيد (ايزوكوئرستين) (Y)، ايزورامتتين r-أ-- روتينوزيد & \\
\hline 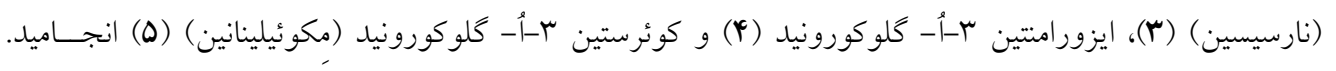 & \\
\hline 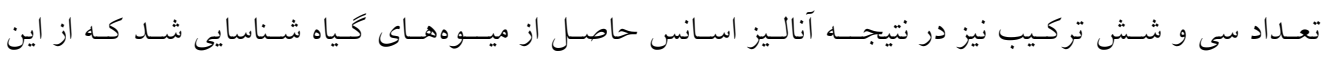 & \\
\hline 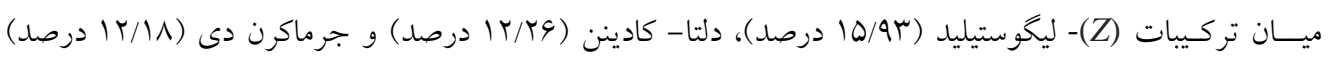 & \\
\hline تركيبات عمده بودند. نتيجه گيرى: نتايج اين مطالعه، ميوههاى كياه كلوس را به عنوان منبعى از كليكوزيدهاى & \\
\hline فلاونوئيدى و مشتقات فتالات معرفى مى كند. حضور اين تركيبات كه خواص بيولوزيك و اثرات سودمند آنها بر & \\
\hline سلامتى در مطالعات بيشين نشان داده شده است، ميوههاى اين كَاه را به عنوان كزينه مناسبى براى مطالعات مرتبط & \\
\hline 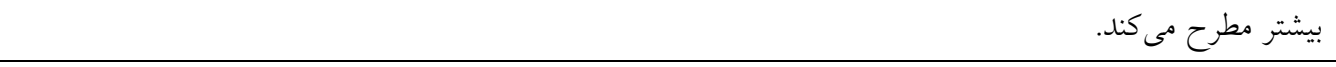 & \\
\hline
\end{tabular}

مخففها: NMR، رزونانس مغناطيسى هستهاى؛ GC-MS، كروماتو گرافى كازى متصل به طيفسنج جرمى؛ TLC، كروماتوكرافى لايه نازى

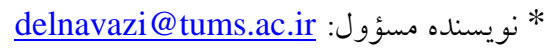
doi: $10.52547 / \mathrm{jmp} .20 .79 .1$

(C) 2020. Open access. This article is distributed under the terms of the Creative Commons Attribution-NonCommercial 4.0 International License (https://creativecommons.org/licenses/by-nc/4.0/) 